\title{
Walter Benjamin en Argentina: una constelación paradójica*
}

\author{
Walter Benjamin in Argentina: a paradoxical constellation
}

\author{
Luis Ignacio García \\ luisggkm@gmail.com \\ (Universidad Nacional de Córdoba, Córdoba, Argentina)
}

\begin{abstract}
Resumen: Este trabajo propone un recorrido panorámico de la presencia de Walter Benjamin en el campo intelectual argentino. Se explicitan primeramente elementos de una teoría política de la recepción planteada en la propia obra de Benjamin. Luego, se organiza el texto a partir de un criterio doble, a la vez histórico y problemático. Las distintas secciones muestran una clara e insistente presencia de Benjamin, a la vez que una limitada producción académica sobre su obra. Cerramos el trabajo con un balance general, sugiriendo las tareas pendientes de la recepción, a la luz de las discusiones teórico-políticas actuales en Argentina y la región.
\end{abstract}

Palabras clave: Walter Benjamin; recepción; traducción; memoria.
Abstract: This work proposes a panoramic study of the presence of Walter Benjamin in the Argentine intellectual field. First, we explain some elements of a political theory of reception set forth in Benjamin's own work. Then, the text is organized based on a double criterion, both historical and problematic. The different sections show a clear and insistent presence of Benjamin, as well as a limited academic production on his work. We close the work with a general balance, suggesting the pending tasks of the reception, in the light of the current theoretical-political discussions in Argentina and the region.

Keywords: Walter Benjamin; reception; translation; memory.

DOI: http://dx.doi.org/10.11606/issn.2318-9800.v25i4p81-98

No se trata de presentar las obras literarias en conexión con su tiempo, sino más bien de hacer evidente, en el tiempo que las vio nacer, el tiempo que las conoce y juzga, o sea, el nuestro.

W. Benjamin

(“Historia de la literatura y ciencia literaria”, 1931)

\section{Introducción: políticas de la transmisión}

Se ha citado hasta el cansancio el famoso pasaje de las tesis "Sobre el concepto de historia" en el que Benjamin enuncia su diagnóstico de una dialéctica

* El presente artículo retoma, desarrolla y actualiza algunos de los resultados de mi tesis doctoral, García (2014), y forma parte de mi trabajo de investigación en el CONICET. Una versión del mismo fue presentada en el II Workshop Interdisciplinar "Teoría Crítica no mundo: America Latina em Foco", Departamento de Filosofía da FFLCH/USP, São Paulo, junio de 2019. 
entre cultura y barbarie. Sin embargo, pocas veces se recuerda el pasaje completo, en el que esa dramática dialéctica que rige la cultura es atribuida específicamente al proceso de transmisión cultural. En efecto, en la séptima tesis se lee: "Jamás se da un documento de cultura sin que lo sea a la vez de la barbarie. E igual que él mismo no está libre de barbarie, tampoco lo está el proceso de transmisión en el que pasa de unas manos a otras" (Benjamin, 1987, p. 182). El problema de la transmisión cultural, de la supervivencia de una obra, el problema de la recepción de un autor, en una palabra, el problema de la tradición, era para Benjamin un problema eminentemente político, tanto porque se ponen allí en juego relaciones de dominación sedimentadas, cuanto porque la memoria histórica afecta decisivamente la colectiva voluntad política de transformación. Aún en el contexto de las citadas tesis, se dice:

El peligro amenaza tanto al patrimonio de la tradición como a los que lo reciben. En ambos casos es uno y el mismo: prestarse a ser instrumento de la clase dominante. En toda época ha de intentarse arrancar la tradición al respectivo conformismo que está a punto de subyugarla (idem, p. 180).

Benjamin pensaba que los procesos de transmisión cultural son ámbitos privilegiados de la lucha de clases. Consideraba que interrumpir el "cortejo triunfal" de la cultura es el objetivo clave de una pedagogía materialista, y que rescatar los materiales olvidados que se alojan, desde "la lejanía de los tiempos”, “como confianza, como coraje, como humor, como astucia, como denuedo" para "construir" la tradición de los oprimidos, es la meta capital del “materialista histórico” (idem, p. 179).

Si a estas consideraciones las encontramos en ese testamento filosófico-político que son las tesis sobre la historia, no llama la atención que el problema de la recepción haya atravesado sostenidamente su itinerario intelectual en distintos momentos y con diversas modulaciones. ${ }^{1}$ De modo que un abordaje sobre la "recepción" de su obra parece justificado desde el propio gesto de la crítica benjaminiana. Y así como Benjamin en "El autor como productor" auspiciaba una activación del público del arte técnico, así también podemos pensar el estudio de la recepción como el estudio de la activación de los legatarios de una cultura, una obra, un autor, destacando el momento de selección y desvío, de la recepción creativa y dislocadora como el momento específicamente político del proceso de transmisión cultural, de insumisión ante la cultura como "patrimonio" de los vencedores. Y entonces podremos interrogar, desde el corazón de la problemática benjaminiana, al lector como productor.

1 El esfuerzo más notable por desarrollar la teoría de la recepción implícita en la obra de Benjamin es el de Garber (1987). 


\section{Arte posaurático y política de masas}

El primer momento de recepción de Benjamin en Argentina está marcado por la labor de un pensador injustamente olvidado: Luis Juan Guerrero (1899-1957), y el contexto histórico-cultural de la "crisis de la cultura". Guerrero perteneció a esa franja intelectual que maduró entre la muerte de Alejandro Korn, en los años 30, y el advenimiento de la generación de la revista Contorno, a mediados de los 50. Un conjunto compuesto de nombres tan relevantes como Saúl Taborda, Carlos Astrada, Francisco Romero, Miguel Ángel Virasoro, Ángel Vasallo, Vicente Fatone, entre otros. A pesar de la gran riqueza de sus producciones, sus representantes fueron rápidamente rubricados bajo el rótulo de la "crítica del positivismo" y del surgimiento de una "nueva sensibilidad" espiritualista e inclinada a la especulación metafísica, soslayándose el estudio de trayectorias y obras en muchos casos notables. Uno de esos casos es el de Luis Juan Guerrero, que con su Estética demuestra que en la generación de la "crítica del positivismo" no todo fue "espiritualismo". Su obra presenta una notable - y tempranísima - inflexión benjaminiana que le permite a la vez participar del clima intelectual de entreguerras sin por ello recaer en la gestualidad orteguiana, tan influyente en aquellos años.

Tras su estancia en Alemania entre 1923 y 1928, en la que realizó su tesis doctoral sobre ética de los valores, Guerrero fue docente de ética, psicología y estética en diversas universidades argentinas. Su trabajo más importante es, sin dudas, su monumental Estética operatoria en sus tres direcciones, preparada durante los largos años de docencia y publicada en tres tomos por Losada, en 1956 los dos primeros, y en 1967, póstumamente, el tercero. Guerrero ya en 1933 incluía en su programa de "Estética" el libro de Benjamin sobre el Romanticismo. Sin embargo, decisivo para su estética fue el trabajo sobre "La obra de arte en la época de su reproductibilidad técnica". Y es de suponer que lo conoció ya en 1936, pues es precisamente en ese año que nuestro autor entabla relaciones con el Instituto de Investigación Social radicado en Nueva York. Entra en contacto directo con Franz Neumann y en relación epistolar con el director del Instituto, Max Horkheimer, con el objetivo de una colaboración académica finalmente frustrada. Dado que en su Estética cita el trabajo benjaminiano de la edición de la Zeitschrift für Sozialforschung, es más que probable que haya sido en ese temprano 1936 que Guerrero accedió al artículo de Benjamin, publicado en francés, precisamente, en el número $\mathrm{V}$ de 1936 de la revista del Instituto.

Ahora bien, ¿cuál es el lugar de Benjamin en la Estética de Guerrero? Para esquematizar una presencia que guarda múltiples matices, que el ensayo sobre la obra de arte de Benjamin ocupa un lugar decisivo en la concepción de Guerrero de (1) la historia del arte, (2) el lugar de la técnica y su relación al arte, (3) el sentido 
político del arte contemporáneo. En cuanto a lo primero, Guerrero abre su Estética con una reflexión sobre el lugar del arte en la historia, planteando el proceso de progresiva desacralización, y apostando a asumir las consecuencias de la definitiva secularización del arte contemporáneo, criticando toda visión compensatoria de una "religión del arte": en Guerrero el arte no encarna ninguna nostalgia por la ausencia de los dioses (tal como se diagnosticaba en el clima heideggeriano de aquellos años), sino sencillamente "el lugar de la ausencia de los dioses" (Guerrero 1956a, 38). En cuanto a lo segundo, Guerrero critica prejuicio anti-tecnológico y apunta, recuperando a Benjamin, que las nuevas formas de reproducción han liquidado la noción tradicional de "autenticidad", y que el estatuto contemporáneo del arte emerge de esa situación. Aquí Guerrero se aproxima al "museo imaginario" de A. Malraux, pero si Guerrero comparte con Malraux su concepción de las transformaciones del arte a partir de la irrupción de la técnica (frente al prejuicio antitécnico de Heidegger), va sin embargo más allá de la restitución del mito burgués del "museo", y de la mano de Benjamin avanza hacia la búsqueda de las consecuencias políticas de la reproductibilidad. Es la función social del arte se transforma decisivamente, y, como dijera Benjamin, el fondo ritual del arte se debe sustituir por un fondo constituido por una práctica otra: la política. De allí que, en tercer lugar, Guerrero postule el tránsito de una estética tradicionalmente centrada en la dimensión contemplativa a una estética decididamente "operatoria", como él la llama, orientada decisivamente por las "demandas" (Guerrero, 1967) de una comunidad histórico-política concreta. Como síntesis de su desarrollo, su estética se cierra, nuevamente citando a Benjamin, en un bello apartado titulado "El cine, primer arte de la vida política en la historia universal". Benjamin, con su "siempre recordado ensayo" (idem, p.238), colabora decisivamente en el planteo de la orientación final de la Estética de Guerrero. En los años del primer peronismo -al que Guerrero prestara un apoyo crítico -, su Estética intenta pensar nada menos que la irrupción de las masas en la vida estética.

\section{Traducción como redención}

Otro rasgo saliente de la paradójica recepción argentina es que, después de este momento materialista y político de la presencia benjaminiana, nos topemos con un ensayista que privilegiará los tonos más marcadamente mesiánico-místicos del pensamiento benjaminiano, precisamente en los años en que, tras la revuelta estudiantil, Benjamin comienza a aparecer en un contexto internacional como profeta de la izquierda radical. Nos referimos a Héctor Álvarez Murena (19231975). La presencia de Benjamin en Murena está atravesada por la cuestión de la traducción. Y lo está en un doble sentido: en el sentido material de que Murena es el primer traductor de Benjamin al castellano, y en el sentido intelectual de que su recepción de la problemática benjaminiana se centra en la concepción del lenguaje 
y de la traducción del berlinés. En efecto, gracias a su mediación, la editorial Sur, dependiente de la revista, lanzó la importante colección “Estudios Alemanes”, del que sólo recordaremos, para nuestros intereses, que durante los años en que participó Murena, se editó buena parte del legado teórico de la escuela de Frankfurt y otros intelectuales afines a Benjamin. En ese marco, aparece en 1967 la traducción, a cargo de Murena, de una colección de ensayos de Benjamin, titulada Ensayos escogidos. Si bien carece de introducción o prólogo de los editores, el volumen incluye en contratapa una ajustada semblanza de Benjamin.

Esta primera traducción castellana resulta comparativamente temprana en el contexto de la recepción internacional, sólo precedida por las ediciones francesa (Oeuvres choisies, Juillard, 1959) e italiana (Angelus Novus, Einaudi, 1962). Pero no sólo fue una edición temprana, sino que además tuvo un impacto efectivo en los primeros pasos de las ediciones castellanas. En efecto, tres de los ensayos editados por Sur formarán parte de la selección editada en Venezuela por Monte Ávila bajo el título Sobre el programa de la filosofía futura y otros ensayos, de mucha difusión. Y a su vez, esta primera versión castellana fue replicada en su totalidad en España por la editorial Edhasa, que sólo le agregó un prólogo de los editores, y la tituló Angelus Novus, en 1971, es decir, el año en que la edición de Benjamin por editorial Taurus recién comenzaba. Sin embargo, este gesto pionero no será continuado en la Argentina por una política coherente y sostenida de edición de Benjamin. ${ }^{2}$

En cuanto a la presencia de Benjamin en la ensayística de Murena, hay que destacar que Murena recupera su filosofía mística del lenguaje. Murena, tras haber practicado una crítica cultural negativa, no materialista, sino más bien fraguada en una crítica de la "civilización fáustica”, de la degradación de la cultura en la era de la tecnocracia y la cultura de masas, que supo enlazar a Martínez Estrada con la Dialéctica del iluminismo, intentó en su último libro de ensayos, La metáfora y lo sagrado (1973), postular una alternativa a la visión fuertemente negativa de sus trabajos anteriores: un concepto de cultura fuertemente matrizado en la concepción benjaminiana del lenguaje y la traducción. Frente a la progresiva totalización de lo social operada por la razón científico-técnica, Murena apuesta a la "metáfora" y la "traducción" como promesa de inscripción de lo otro en el lenguaje humano, como apertura de sentido amparada en el fondo insondable, sagrado, que preside el acto de traducción y testimonia la fragilidad de la propia historia humana.

Jamás existirá la versión definitiva de un libro a ningún idioma. Nunca se terminó, se terminará, de traducir libro alguno. Esto exige preguntar: ¿qué es lo absolutamente intraducible que permite y reclama la posibilidad y la práctica infinitas de traducción? Lo absolutamente intraducible es esa Unidad perdida, que la traducción recuerda con su incesante esfuerzo por reunir las cosas convirtiendo unas en otras (Murena, 2002, p. 447).

2 A lo largo de más de cuarenta años, sólo se agregarán las siguientes ediciones locales: Benjamin 1974, 1989 y 2009. 
Tal la benjaminiana tarea (-fracaso) del traductor según Murena. Esta voz, totalmente anacrónica en la Argentina de 1973, intentaba plantear una respuesta, desde el limitado horizonte de una Kulturkritik espiritualista, a la uniformización de la sociedad de masas, y parece dar cuenta de la espiral de violencia política (en la esotérica lectura de Murena, consecuencia de la hybris de Babel) que asolaba al país en esos años. La voz de Murena tendrá muy escaso eco en aquel entonces, pero será recuperada en nuestros días, en parte a la luz de la recuperación del Benjamin místico del lenguaje.

\section{Compromiso o vanguardia}

Desde una matriz estética y política totalmente diversa, el joven Ricardo Piglia (1940-2017) se valdrá de algunos aspectos de la crítica benjaminiana, mucho más a tono con la recepción internacional del momento, que volvía sobre un Benjamin marxista, brechtiano, y anti-frankfurtiano. En algún sentido, y de un modo más militante pero con alcance teórico mucho menos ambicioso, volvía al Benjamin que le interesó a Guerrero, aunque sin ninguna referencia a este último. A lo largo de la década del 70, Piglia irá construyendo los parámetros de su crítica de la mano de los formalistas rusos y de Brecht, y es en ese marco que ingresará su acotada recepción de Walter Benjamin. El propio Piglia lo plantea en diversas intervenciones retrospectivas posteriores: se trataba

de intervenir en el debate de la izquierda, enfrentar la tradición de Lukács y el realismo, empezar a hacer entrar los problemas que planteaban Brecht, Benjamin, la tradición de la vanguardia rusa de los años 20, Tretiakov, Tinianov, Lissitsky (Piglia, 1986, p. 103).

Piglia inscribe a Brecht y Benjamin en el contexto de los debates sobre compromiso y vanguardia, característicos en toda Latinoamérica en aquellos años de fuerte politización. Construye así un Benjamin que permite conciliar los reclamos de articular vanguardia política y vanguardia estética en un mismo programa estéticopolítico: en una "estética de la producción". Según Piglia, pensar a la cultura como rama de la producción material de la sociedad -meollo de una tal estética de la producción -, permitiría ir más allá de la escisión entre cultura y política, presente no sólo en las estéticas idealistas, sino también de las estéticas marxistas "humanistas" que tanta influencia tuvieron en nuestro país y también en el debate internacional a lo largo de los años 60, sobre todo a través del "compromiso" sartreano o del "realismo" lukacsiano. En las estéticas "humanistas", según Piglia, pueden convivir sin problemas opiniones políticas izquierdistas con prácticas artística burguesas, una escisión patente, por ejemplo, en el paradigmático rechazo de la vanguardia por parte de Lukács. Eso no sería sino una consecuencia de seguir pensando al arte - 
desde las matrices burguesas y "humanistas" del idealismo alemán - como expresión del espíritu y no como parte del proceso material de producción. Contra ello, Piglia recupera al Benjamin de "El autor como productor":

Como había planteado Walter Benjamin: “En lugar de preguntarse cuál es la posición de una obra en relación con las condiciones de producción de una época, hay que preguntarse cuál es su posición en el interior de esas condiciones de producción. Esta pregunta afronta directamente la función que tiene una obra en el interior de esas relaciones de producción" (Piglia, 1975, p.7).

Así, si el arte es una rama de la producción material de la sociedad, se puede reivindicar su especificidad (legitimándose así la experimentación estética), a la vez que pasa a tener un sentido político, ahora inmanente. De este modo se intenta enfrentar la tradición del realismo (que no sólo se refiere a su visión de Lukács sino también a la generación de escritores argentinos anterior, de Contorno y El escarabajo de oro, y del boom latinoamericano en general) y articular así el compromiso político con la experimentación estética.

También se pueden encontrar referencias directas o alusiones a Benjamin en la propia narrativa pigliana. Y si tenemos en cuenta la fuerte influencia de su obra, narrativa y crítica, en los años 80 , a pesar de no haber escrito casi sobre Benjamin, sus breves comentarios tuvieron cierto impacto en la siguiente etapa de recepción, posterior a la dictadura.

\section{Modernidades alternativas en disputa}

De hecho, fue en el ámbito de la revista Punto de Vista, de la que Piglia participara en sus primeros números, uno de los principales focos de difusión de Benjamin en los años 80. Con todo, la producción sobre Benjamin en los 80 es aún muy magra. Su nombre circulaba asiduamente pero sin cristalizar en lecturas críticas de su obra. En realidad, la recepción más productiva recién se producirá a partir del Simposio de 1992, al que luego nos referiremos.

Estamos ahora en los años de la posdictadura. La "crisis del marxismo" y la irrupción de las temáticas de la "modernidad" y su "crisis" marcan la época. Libros como Viena Fin-de-Siècle de Carl Schorske o Todo lo sólido se desvanece en el aire. La experiencia de la modernidad, de Marshall Berman, serán el marco en el que ingresará un Benjamin teórico de la modernidad, fisognomista de la gran ciudad, teórico de la experiencia. Una modernidad periférica: Buenos Aires 1920 y 1930, de Beatriz Sarlo (directora de Punto de Vista), de 1988, es un influyente producto de este clima que, apenas mencionando a Benjamin como inspirador en su prólogo, marca sin embargo el signo de una época, y que pudo ser leído en paralelo al tipo de indagación de "El París del Segundo Imperio en Baudelaire". Los trabajos sobre 
Baudelaire serán el eje de este tipo de lectura, a los que se sumará una incipiente lectura de los Passagen.

En este contexto es de destacar un intercambio polémico que se publicó en la revista Punto de Vista, y que ya en 1988 anticipa dos tipos influyentes de lectura de Benjamin a partir de entonces en nuestro país. El cruce se produjo entre Ricardo Forster, por un lado, y Anahí Ballent, Adrián Gorelik y Graciela Silvestri, por el otro (Forster, 1987, 1988; Ballent, Gorelik, Silvestri, 1988a, 1988b). El debate tendrá como eje la pregunta por la modernidad y su relación con la ciudad. Forster, en su artículo inicial, se esfuerza por destacar, tal como lo hará sostenidamente a lo largo de su itinerario, los "claroscuros de la modernidad", proponiendo una dialéctica en el seno de las vanguardias de principios de siglo entre una tendencia racionalista, funcionalista y modernizadora, y una "dimensión onírica, nocturna y arcaizante", en la que ve aflorar "viejos temas románticos", o "símbolos de perfiles míticos". Diagnostica en el devenir urbano subsiguiente el primado de la versión racionalista e ingenieril, denunciando la "mecanización del arte y de la vida", la "descomposición" y "deshumanización" de la ciudad en la "metrópolis" contemporánea, y proponiendo provocativamente en el final de su ensayo: "Frente al desencantamiento del mundo provocado por la modernidad secularizadora es posible, siempre desde la perspectiva de la resistencia, aportar a su reencantamiento, posar la mirada en otro horizonte" (Forster, 1987, p.10). El Benjamin teórico de la experiencia urbana ya aparece en este ensayo, junto a Simmel, en la lectura de Cacciari, junto al ya citado Berman, y en un contexto embebido de la problemática de la Viena fin de siècle. Lo que más nos interesa ahora es que ya aquí vemos las claves de la lectura que Forster comienza a diseñar de Benjamin, y que cristalizarán en sus libros posteriores: no tanto Benjamin por Benjamin mismo, sino recuperando un tramo particularmente intenso del pensamiento de entreguerras y una combinación deliberadamente ecléctica de sus principales representantes; un Benjamin ajeno al marxismo militante de los 70, lejano ya al "plumpes Denken" de Brecht, y mucho más próximo una Kulturkritik negativa en clave ensayística; la presencia de la dimensión "romántica", "mítica", que luego cobrará espesor en su sostenida tematización de la dimensión judía del pensamiento benjaminiano.

La crítica de Ballent, Gorelik y Silvestri, para lo que a nosotros nos interesa, se plantea desde un mismo registro de legitimidad, esto es, desde "el trabajo de la escuela de Venecia a partir del 'descubrimiento' de Benjamin y la 'Gran Viena'”, pero plantea fuertes objeciones al planteo de Forster: a) la crítica de la idea de "dos modernidades", o mejor, de la dualidad entre "racionalización" y "mito", y el esfuerzo por una visión más matizada y diferenciadora de lo moderno, lo urbano y las vanguardias; b) la crítica del uso "romántico" de la producción teórica de la "escuela de Venecia", crítica, según los articulistas, de ese sesgo "anti-metropolitano" de 
Forster; c) la crítica de la Kulturkritik meramente negativa, y su prejuicio antimodernizador, anti-tecnológico, que no ve el costado "positivo" y "constructivo" de la vanguardia. Todo ello desde la reivindicación de una mirada histórica (en implícita polémica con la perspectiva ensayístico-filosófica del criticado) que permita pensar la ciudad real, incluso desde una perspectiva de intervención concreta sobre la planificación urbana de Buenos Aires.

Estas dos líneas de lectura son anticipatorias no sólo de la recepción posterior de Benjamin, sino también del propio devenir de una franja importante de la historia intelectual argentina de los últimos años, reflejando dos actitudes diversas ante la Argentina posdictatorial: por un lado, el espacio democrático-progresista, anclado en una defensa del modernismo estético y de la ilustración filosófica, de Punto de Vista, y por otro, la crítica cultural negativa, el ensayismo antiprogresista, de tintes romantizantes, sensible a la dimensión no iluminista de lo moderno, a lo religioso y lo mítico, y a la dimensión no democrática de lo político, que en los años 90 encuentra un espacio en la revista Confines (luego Pensamiento de los confines), con el propio Forster, además de Nicolás Casullo y Alejandro Kaufman como principales animadores.

Pero tampoco pueden perderse de vista los fuertes puntos de contacto entre ambas lecturas, que dan cuenta de un zócalo ideológico de época: por un lado, el eje de la recepción de Benjamin es el problema de lo moderno y la ciudad, Schorske y Berman son referencias indiscutidas; por otro, el Benjamin marxista, brechtiano, el teórico de la condición del intelectual bajo el capitalismo, apenas si aparece en esta etapa de recepción. En el próximo apartado veremos las excepciones a este consenso finalmente "posmarxista". Pues, en efecto, el anti-progresismo de Forster no repone el "materialismo histórico" benjaminiano, sino la dialéctica de catástrofe y redención, ese "mesianismo" que si en los años 90 lo lleva a través del descubrimiento del judaísmo y del "anticapitalismo romántico", en la década siguiente aproxima a Forster a los "populismos" latinoamericanos, ajenos al progresismo democráticoiluminista de sus contrincantes. Y a su vez, si los ensayistas de Punto de Vista recuerdan, con justicia, la proximidad de Benjamin con el ímpetu tecnológico y modernizador de la vanguardia histórica, no lo hacen, como Benjamin, vinculando ese modernismo intrínsecamente con los intereses militantemente emancipatorios del singular marxismo benjaminiano. Nos recuerdan, acertadamente, que Benjamin quiso "comprender juntos Breton y Le Corbusier", pero olvidan que el espacio en el que esa articulación se hacía posible era el "materialismo histórico" benjaminiano y sus categorías clave, como "fetichismo de la mercancía", "montaje" o "imagen dialéctica".

En este contexto se publica en la Argentina el primer libro dedicado a Benjamin: W. Benjamin - Th. W. Adorno: el ensayo como filosofía, de Ricardo Forster, de 1991. 
El libro desarrolla la línea de lectura antes sugerida, con un acento cada vez más marcado en las relaciones entre Benjamin y el judaísmo. Los principales parámetros de la lectura de Forster serán Gershom Scholem y Hanna Arendt, George Steiner y Elías Canetti. Consecuentemente, se privilegia el Benjamin cabalista de Scholem, el homme de lettres y "pensador poético" de Arendt, el teólogo del lenguaje de Steiner, el clima mitteleuropa de Canetti. Aparece marcadamente la figura del pensador del exilio (más en el sentido de la diáspora que en el de la precarización material del intelectual), y los textos más convocados serán, lógicamente, los ensayos sobre el lenguaje y las tesis "Sobre el concepto de historia". En algún sentido, Forster regresa al tono de lectura de Murena (no es un azar que Confines haya contribuido a la relectura de Murena).

No nos sorprenderá que un artículo de Ballent, Gorelik y Silvestri dos años posterior, "Las metrópolis de Benjamin" (1993), nos encontremos con un diagnóstico crítico, precisamente, de ese Benjamin que Forster construía en su libro y que luego el grupo de Confines mostrará en su revista. Con un ojo en los últimos desarrollos (posmarxistas) de los italianos M. Cacciari y F. Dal Co (otrora destacados miembros de la escuela de Venecia) y el otro puesto en las lecturas locales como la de Forster, critican un "clima heideggeriano que recupera el intento de salir del pensamiento dialéctico-constructivo (...). Y así se llega a una acepción, débil y no trascendente, de lo místico" (1993, p.27). Denuncian que reuniendo una serie de "motivos (el desplazamiento de Benjamin desde la variada constelación del marxismo occidental al pensamiento original de los judíos de la Europa central; y la atención a un lenguaje metafórico, cuya función ya no es ilustrativa o argumentativa, sino sustantiva) aparece el nuevo Benjamin para la cultura urbana."

Esta doble tendencia ("comentadores" y "partidarios", "modernistas" y "críticos de lo moderno", "progresistas" y "románticos") determinará en parte la producción sobre Benjamin posterior. Así, en la línea de los "comentadores" pueden destacarse dos trabajos vinculados al círculo de Punto de Vista: el muy informado y lúcido ensayo de Ricardo Ibarlucía sobre Benjamin y el surrealismo (Ibarlucía, 1998), o el prolijo ensayo del escritor Martín Kohan sobre Benjamin y "las ciudades" (2004). La otra línea se desplegará en los múltiples libros de Forster, ${ }^{3}$ y en el ámbito de la revista Confines, sobre todo en las apropiaciones de Casullo y Kaufman, muy relevantes para la difusión de esta lectura "mitteleuropea" de Benjamin. ${ }^{4}$

En este contexto de discusión se realiza en octubre de 1992, en el GoetheInstitut de Buenos Aires y a instancias de Nicolás Casullo, un simposio internacional sobre Walter Benjamin, con motivo del centenario de su nacimiento. A partir del congreso se publicó un importante volumen que reúne los trabajos presentados

3 Véase sobre todo Forster, 2001, 2005, 2009a, 2009b.

4 Véase Casullo, Forster, Kaufman, 1999. 
(VVAA, 1993). La importancia de este encuentro es difícil de exagerar, y se la puede analizar destacando tres de sus logros fundamentales: haber reunido a los principales investigadores sobre Benjamin de nuestro país; haber incluido a protagonistas fundamentales de la recepción latinoamericana, sobre todo la tan dinámica recepción brasilera; haber invitados a destacados investigadores benjaminianos alemanes. Desgraciadamente, esta cuidadosa atención al ámbito internacional de la recepción benjaminiana desaparece hasta tiempos muy recientes. ${ }^{5}$ En cualquier caso, no caben dudas de que con este Simposio comienza una nueva etapa, de recepción más sistemática y rigurosa de la obra de Walter Benjamin en nuestro país.

\section{Un marxismo de la adversidad}

Una línea menos visible en la escena intelectual argentina, pero no menos relevante, se esforzó por recuperar activamente el trasfondo marxista del pensamiento benjaminiano. En todos los casos, se destaca la singularidad, excepcionalidad, o heterodoxia benjaminiana. Pero también en todos los casos, aparece Benjamin como un modo de salvar el marxismo en la era de la crisis terminal de sus versiones clásicas, ortodoxas o dogmáticas. De ese modo se perfilan los trazos de lo que Sazbón llamara, en uno de sus exquisitos ensayos, un "marxismo de la adversidad", esto es, un pensamiento que, consciente de la catástrofe, intenta aún construir una perspectiva emancipatoria desde el horizonte (profano) del legado de Karl Marx.

La lectura de Benjamin por parte de José Aricó (1931-1991) encierra cierta paradoja: Aricó fue de los pocos que insistirán marcadamente en el costado marxista del pensamiento benjaminiano, pero a la vez su descubrimiento de Benjamin se produce los mismos años en que se compromete con la recuperación del socialismo revisionista y socialdemócrata que Benjamin tanto criticara. Con todo, será Aricó quien recogerá del modo más ejemplar en nuestro país el reclamo benjaminiano de un rescate de la tradición de los oprimidos. La dispersa presencia de Benjamin en sus escritos coincide con su formulación de en un verdadero programa de una historia del marxismo latinoamericano que comienza a plantear desde fines de los años 70, con sus trabajos sobre José Carlos Mariátegui. De hecho, será en sus textos sobre el peruano donde más asiduamente aparece el nombre de Benjamin, otro soreliano crítico del marxismo mecanicista. En esa suerte de autobiografía intelectual que es La cola del diablo aparece, ya sin siquiera citarlo, una declaración de fe benjaminiana que resume toda la actividad intelectual ariqueana desde fines de los 70 hasta su muerte:

5 En los que dos importantes compilaciones recuperan los lazos con la recepción latinoamericana y alemana: Buchenhorst, Vedda, 2008, y Vedda, 2008. 
La tarea entonces no puede ser otra que arrancar el pasado de la tradición en la que las ideologías dominantes lo han aprisionado. Desde esta perspectiva nunca nada se ha perdido para siempre; quien esté dispuesto a hacer saltar el continuum de la historia no puede aceptar la trivial creencia en el progreso de la cultura (Aricó, 1988, p. 16).

Tal el sentido de su recuperación de la figura de Mariátegui, su rescate de Juan B. Justo, y sus múltiples formulaciones sobre las alternativas de un "marxismo latinoamericano".6

Sin embargo, Benjamin es para Aricó un inspirador fundamental de los últimos años de su labor, pero no un objeto de estudio. Quien escribió ensayos muy importantes sobre Benjamin intentando plantear los alcances de su relación con Marx y con el marxismo es el recientemente fallecido José Sazbón (1937-2009). ${ }^{7}$ Tras destacar la singularidad de la apropiación del marxismo por parte de Benjamin, plantea Sazbón:

La dimensión más general y sistemática de aproximación entre Marx y Benjamin - así como la que permite apreciar la "contribución" de este último al marxismo - surge de la siguiente premisa: en su tarea de profundización del materialismo histórico, Benjamin, en su Libro sobre los Pasajes, habría buscado configurar la superestructura cultural del capitalismo como un complemento de la elaboración, por Marx, de las articulaciones de su estructura económica (Sazbón, 2002, p. 158).

A partir de esta premisa, Sazbón desenvuelve las miradas de ambos en torno a una diversidad de tópicos de la tradición marxista: la relación estructura/superestructura, la revolución, la historia, la utopía, el movimiento obrero, el fetichismo de la mercancía, la relación entre producción y circulación, etc. La sobria y cristalina escritura de Sazbón evita la (tan frecuente) lectura admirativa, y plantea también con claridad los límites de la aproximación entre Marx y Benjamin. En otro de sus trabajos contrasta los proyectos intelectuales de ambos, señalando:

El de Marx suponía una apuesta a la coordinación positiva de ilustración científica y voluntad emancipatoria. Benjamin, en cambio, testigo de la declinación de esa promesa, confió más bien en un brusco despertar mesiánico y en una recuperación de la energía utópica del pasado (Sazbón, 2009, p. 286).

Frente a Marx, destaca Sazbón de Benjamin "su sombría certidumbre de la precariedad del canon ilustrado en una época de barbarie triunfante” (idem, p. 287), y remata: "Se diseña así un marxismo de la adversidad, abismalmente consciente de los espejismos que generaron su parálisis y de las servidumbres que fomentaron su

6 Además, Aricó prepara un suplemento especial para La ciudad futura dedicado a Benjamin: La ciudad Futura, $\mathrm{n}^{\circ}$ 25-26, 1990-1991, Suplemento/9, que incluye dos intervenciones de Aricó: "Walter Benjamin, el aguafiestas", y, junto a Marcelo Leiras, "Benjamin en español".

7 Entre sus trabajos habría que destacar "Historia y paradigmas en Marx y Benjamin" y "La historia en las 'Tesis' de Benjamin: problemas de interpretación”, en Sazbón, 2002, y “El legado teórico de la Escuela de Frankfurt”, "Historia y experiencia” e "Historia intelectual y teoría crítica”, en Sazbón, 2009. 
impotencia" (idem). Como Aricó en el ámbito de la historiografía de las izquierdas, Sazbón en un dominio teórico-filosófico nos ofrece no sólo una matriz desde donde pensar las relaciones entre Benjamin y el marxismo, inscribiéndolo certeramente en el ámbito del marxismo occidental, sino además la apuesta por un marxismo crítico en un contexto histórico de máxima adversidad ideológico-política. Si Aricó nos legó un amplio programa -benjaminiano - de rescate de la tradición de los oprimidos en Latinoamérica, Sazbón nos dejó un modelo de labor crítica que aúna el rigor y la sobriedad con una inextinta exigencia emancipatoria.

\section{Memoria y experiencia: Benjamin en la ESMA}

Sin embargo, uno de los ámbitos en que la presencia de Benjamin se ha tornado más intensa en el último tiempo es la arena de discusiones en torno a la "memoria". Discusiones que, como se sabe, se intensificaron después de mediados de los años 90, y, sobre todo, en los últimos años, en los que diversas coyunturas políticas y culturales marcaron un verdadero auge de la memoria en nuestro país. En los años de la "recuperación" de los centros clandestinos de detención y de la reapertura de los juicios contra los genocidas, la "memoria" marca el signo de la más reciente recepción de Benjamin en nuestro país.

En este contexto, ya no serán ni el Benjamin vanguardista, ni Benjamin teórico de lo moderno, ni el Benjamin marxista los que prevalecerán. El interés se centra, más bien, en el teórico de la experiencia y, más en particular, del ocaso de la experiencia. Ahora, Benjamin no entra ya más en sintonía con las ciudades de Schorske o Berman, ni con las tradiciones de Marx o la escuela de Frankfurt (ni mucho menos con los viejos tópicos de Malraux o Brecht), sino con Giorgio Agamben o Paul Ricoeur, con Andreas Huyssen o Georges Didi-Huberman. Ya no se recuperan tanto los textos sobre Baudelaire, ni la fisognomía urbana de los Passagen, sino trabajos como "El narrador" o "Experiencia y pobreza", y si las tesis "Sobre el concepto de historia" pudieron ser leídas como un manifiesto de marxismo crítico, ahora son mucho más una melancólica teoría de la rememoración. Desaparecen el flâneur y el París del Segundo Imperio y emergen los conceptos de ruina, "mémoire involontaire", y la problemática de Auschwitz. En este contexto cobran nueva legitimidad los tópicos del Benjamin judío.

De este nuevo contexto, en el que Benjamin aparece una y otra vez, de manera recurrente y diseminada, sólo quisiéramos mencionar dos emergentes de importancia. Por un lado, el modo en que Beatriz Sarlo sitúa a Benjamin en el centro de uno de los debates que, en torno a la problemática de la memoria, ha cobrado mayor rispidez: el debate sobre el testimonio y el lugar de la experiencia subjetiva. En un libro polémico, Sarlo denuncia un "giro subjetivo" en la cultura 
contemporánea, criticando, a partir de la convicción de que "es más importante entender que recordar", una "cultura de la memoria" que sitúa a la experiencia personal como argumento de verdad. En ese contexto Benjamin aparece como quien planteara de manera cabal las aporías de la relación entre historia y memoria. Sarlo propone una tensión entre "El narrador" y las tesis "Sobre el concepto de historia": si el primero diagnostica "la disolución de la experiencia y del relato que ha perdido la verdad presencia antes anclada en el cuerpo y la voz" (Sarlo, 2005, p. 34), en las segundas Benjamin criticaría la cosificación del positivismo histórico, que anula la relación de la verdad histórica con la subjetividad que la experimenta, apostando a la redención del pasado por la memoria. Según Sarlo, lo que en Benjamin permanecía como aporía desgarradora, una aporía que lo condujo a la apuesta desesperada por lo mesiánico, en el presente de nuestra "cultura de la memoria" es tomado con la liviandad de una disponibilidad experiencial del pasado que el propio Benjamin rechazó.

Según Benjamin, aquello que fue posible hasta un momento determinado de la historia se volvió imposible, a causa del carácter irreversible de la intervención capitalista moderna sobre la subjetividad; pero hoy, incluso citando a Benjamin, la restauración de un relato significativo de la experiencia se considera posible, pasando por alto precisamente aquello que, para Benjamin, volvía trágica la situación contemporánea (Sarlo, 2005, pp. 50-51). ${ }^{8}$

De un modo muy polémico, y cuestionable, Sarlo polemiza con la actual pretensión de verdad del testimonio que impregna una amplia serie de intervenciones políticas y culturales.

Por su parte, un importante libro sobre las formas de reconstrucción del pasado reciente dedica la mitad de su extensión a problematizar "pensamientos sobre la memoria y la historia", ofreciendo, en una serie de amplios artículos relativamente independientes, presentaciones al pensamiento de Marx, Nietzsche, Benjamin, Koselleck, Ricoeur y Agamben sobre la problemática. Bajo el título "Benjamin o la cita revolucionaria con el pasado" se recupera fuertemente el sentido político de la concepción benjaminiana de la memoria. De este modo, Benjamin ingresa en un debate singular, en una mesa de discusión junto a una selección singular de pensadores, y lo hace en el formato de un libro de divulgación que pretende instalar las coordenadas de un debate político sobre el pasado reciente. Ya en la propia introducción al libro muestran los autores el talante benjaminiano de la problemática en juego en la Argentina contemporánea: "La memoria, los testimonios, representan los intentos por conjurar el carácter intransmisible de la experiencia vivida en la modernidad tardía" (Oberti y Pittaluga, 2006, p. 23).

8 Véanse Vallina, 2009; Kaufman, 2006, que incluye una crítica a su lectura de Benjamin que prolonga el contrapunto planteado en nuestro apartado IV. 
En octubre de 2010 tuvo lugar un simposio titulado "Recordando a Walter Benjamin. Justicia, Historia y Verdad. Escrituras de la Memoria”, en la ex-ESMA (el campo de concentración y exterminio más grande de la última dictadura militar, recuperado para los organismos de derechos humanos en 2004, por decisión del presidente Néstor Kirchner). Si el simposio, en pleno auge del desarrollo de los juicios contra los genocidas, vino a consolidar la centralidad de Benjamin en los debates sobre la memoria en la Argentina, un episodio dramático hizo irrumpir la historia en nuestro encuentro: la sorpresiva muerte de Néstor Kirchner en medio de las fechas del simposio obligó a reprogramar todas las actividades, con sus cientos de participantes de todo el mundo, aplazándolas en dos días, e impregnando de manera decisiva el despliegue de nuestras subsecuentes discusiones. En 2013 se publicó un libro con una selección de intervenciones del Simposio. El título es impactante y elocuente: Walter Benjamin en la ex Esma (Jozami, Kaufman, Vedda, 2013)

Benjamin quedaba así definitivamente incorporado a nuestra historia reciente.

\section{Coda: (r)excepción}

Este recorrido nos permite afirmar que la recepción de Walter Benjamin en la Argentina ha sido singular y paradójica. Próxima y en parte dependiente de la recepción internacional, sin embargo ostenta marcas que le otorgan un carácter particular. La "constelación austral" del pensamiento benjaminiano ha sido pionera y extendida, a la vez que magra e intermitente. El primer impacto de Benjamin en nuestra historia intelectual se puede reconocer en años tempranísimos, en mucho anteriores a los de su recepción internacional, pero sin embargo el primer libro a él dedicado se publica recién en 1991. La edición internacional de Benjamin tiene un hito precursor en la Argentina, con la publicación en 1967 de sus Ensayos escogidos, pero sin embargo la historia de ediciones de Benjamin en la Argentina apenas cuenta, hasta la fecha, con cuatro libros del berlinés editados en el país. Por último, Benjamin se convirtió con los años en una moda apasionante para la intelectualidad de izquierda, incluso se ha criticado ese boom, pidiéndose hasta un "olvidar a Benjamin" (Sarlo, 1995), pero, sin embargo, la efectiva producción sobre Benjamin en la Argentina es bastante magra, aún hoy, incluso comparada con la recepción en otros países no centrales. El objetivo de este texto fue construir en sus trazos más generales los perfiles del proceso de recepción de Benjamin en la Argentina, a los fines de rescatar y valorar lo hecho, y de calibrar lo que queda por hacer.

Nuestro recorrido termina en los primeros años del nuevo siglo, esto es, en el apogeo del reciente período de hegemonía de gobiernos populares en América Latina. Pero sabemos que hoy somos testigos de una nueva fase neoconservadora que nos impone nuevos (y violentos) desafíos. En agosto de 2018, y en vistas de las 
nuevas formas de persecución y acoso judicial y mediático de la oposición política en toda la región, organizaciones de derechos humanos en Argentina publicaron una declaración en la que diagnostican críticamente la nueva situación y declaran: "Denunciamos que la Argentina está sometida a un estado de excepción permanente que permite el atropello y la violación de los derechos del pueblo". ${ }^{9}$ La alusión, implícita pero clara, a la octava tesis "Sobre el concepto de historia" muestra que, aunque académicamente endeble, la recepción cultural y política de Benjamin en Argentina sigue siendo intensa. $Y$ además parece dejar planteado que una nueva fase se abre, después del auge de los debates sobre la memoria, y en la dirección de la interrogación en torno a ese "estado de excepción" que ha devenido la regla para los oprimidos, para el "campo popular" en la Argentina y en la región, un nuevo tipo de "estado de excepción" que se expresa en manifestaciones mucho más sutiles que las tradicionales de los golpes de estado. Para usar los términos de "Hacia la crítica de la violencia": si las dictaduras militares asumieron el rol de la "violencia fundacional" del neoliberalismo en América Latina, la "violencia conservadora" del neoliberalismo actual en crisis no es la misma ciertamente, aunque participe del mismo ciclo neoliberal. La interrogación por ese "verdadero estado de excepción" que pudiera oponerse a esta siniestra excepción devenida regla (gracias a los medios y al poder judicial) parece ser el sol que guiará el nuevo heliotropismo en torno a la obra de Benjamin en América Latina.

\section{Referencias}

Aricó, J. M. (1988). La cola del diablo. Itinerario de Gramsci en América Latina. Caracas: Nueva Sociedad.

Ballent, A., Gorelik, A., \& Silvestri, G. (1988a). Ante las puertas de la ciudad. Zaratustra o su mono. Punto de Vista 32, 17-24.

Ballent, A., Gorelik, A., \& Silvestri, G. (1988b). Vanguardias: filosofía e historia. Punto de Vista 33, 48.

Ballent, A., Gorelik, A., \& Silvestri, G. (1993). Las metrópolis de Benjamin. Punto de Vista 45, 19-27.

Benjamin, W. (1987). "Tesis de filosofía de la historia”. In: Discursos interrumpidos I. Prólogo. Traducción y notas de J. Aguirre. Madrid: Taurus.

Benjamin, W. (1974). Reflexiones sobre niños, juguetes, libros infantiles, jóvenes y educación. Traducción de Juan J. Thomas. Bs. As.: Nueva Visión. (Reed. aumentada en 1989.)

Benjamin, W. (1992). Cuadros de un pensamiento. Selección, cronología y postfacio de Adriana Mancini, traducción de Susana Mayer (con la colaboración de A. Mancini). Bs. As.: Imago Mundi.

9 Abuelas de Plaza de Mayo, "El país está sometido a un estado de excepción permanente", en https://www.abuelas.org.ar/noticia/la-argentina-estl-sometida-a-un-estado-de-excepcion-1031, 2018 [acceso en 27.11.2020]. 
Benjamin, W. (2009). Estética y política. Traducción de Tomás J. Bartoletti y Julián Fava, introducción de Ralph Buchenhorst. Bs. As.: Las Cuarenta.

Buchenhorst, R. y Vedda, M. (eds.) (2008). Observaciones urbanas: Walter Benjamin y las nuevas ciudades. Bs. As.: Gorla.

Casullo, N., Forster, R., \& Kaufman, A. (1999). Itinerarios de la modernidad. Bs. As.: Eudeba.

Forster, R. (1987). Perplejidades de la modernidad. Punto de Vista, 31, 6-10.

Forster, R. (1988). Las otras puertas del burdel. Punto de Vista, 33, 40-47.

Forster, R. (2001). Walter Benjamin y el problema del mal. Bs. As.: Altamira.

Forster, R. (2008). “Lecturas de Benjamin: entre el anacronismo y la actualidad”. In: Buchenhorst, R. y Vedda, M. (eds.). Observaciones urbanas: Walter Benjamin y las nuevas ciudades. Bs. As.: Gorla.

Forster, R. (2009). Los hermeneutas de la noche. De Walter Benjamin a Paul Celan. Madrid: Trotta.

Forster, R. (2009). Benjamin: una introducción. Bs. As.: Quadrata/Biblioteca Nacional.

Garber, K. (1987). Rezeption und Rettung. Drei Studien zu Walter Benjamin. Tübingen: Niemeyer.

Garcia, L. I. (2008). La escuela de Frankfurt en Sur. Condiciones y derivaciones de un incidente editorial. Sociedad 27.

Garcia, L. I. (2009). Entretelones de una "estética operatoria”. Luis Juan Guerrero y Walter Benjamin. Prismas. Revista de Historia Intelectual, 13(1), 89-114.

Garcia, L. I. (2014). Modernidad, cultura y crítica. La escuela de Frankfurt en Argentina (1936-1983). Córdoba: Universidad Nacional de Córdoba. Disponible en https://ffyh.unc.edu.ar/boletin/ediciones_anteriores/archivos/imagenes/e-books/EBOOK_GARCIA.pdf

Guerrero, L. J. (1956a). Estética operatoria en sus tres direcciones. I. Revelación y acogimiento de la obra de arte. Estética de las manifestaciones artísticas. Bs. As.: Losada. (Hay una reedición del 2008 en editorial Las cuarenta y Biblioteca Nacional).

Guerrero, L. J. (1956b). Estética operatoria en sus tres direcciones. II. Creación y ejecución de la obra de arte. Estética de las potencias artísticas. Bs. As.: Losada.

Guerrero, L. J. (1967). Estética operatoria en sus tres direcciones. III. Promoción y requerimiento de la obra de arte. Estética de las tareas artísticas. Bs. As.: Losada.

Ibarlucía, R. (1998). Onirokitsch. Walter Benjamin y el surrealismo. Bs. As.: Manantial.

Jozami, E., Kaufman, A., \& Vedda, M. (2013). Walter Benjamin en la Ex ESMA. Bs. As.: Prometeo.

Kaufman, A. (2006). Aduanas de la memoria. A propósito de Tiempo pasado de Beatriz Sarlo. Zigurat, 6, UBA.

Kohan, M. (2004). Zona urbana. Ensayo de lectura sobre Walter Benjamin. Bs. As.: Norma. 
Murena, H. A. (2002). "La metáfora y lo sagrado [1973]". In: Visiones de Babel. México: FCE.

Oberti, A. , \& Pittaluga, R. (2006). Memorias en montaje. Escrituras de la militancia y pensamientos sobre la historia. Bs. As.: El cielo por asalto.

Piglia, R. (1975). Notas sobre Brecht. Los Libros, 40.

Piglia, R. (1986). “Novela y utopía”. In: Crítica y ficción. Buenos Aires: Planeta/Seix Barral.

Sarlo, B. (1995). Olvidar a Benjamin. Punto de Vista, 53,16-19.

Sarlo, B. (2000). “Lectores: comentaristas y partidarios". In: Siete ensayos sobre Walter Benjamin. Buenos Aires: FCE.

Sarlo, B. (2005). Tiempo pasado. Cultura de la memoria y giro subjetivo. Una discusión. Bs. As.: Siglo XXI.

Sazbón, J. (2002). Historia y representación. Bs. As.: UNQ.

Sazbón, J. (2009). Nietzsche en Francia y otros estudios de historia intelectual. Bs. As.: UNQ.

Traine, M. (1994). Los vínculos del "Instituto de Investigaciones Sociales" de Frankfort con la Universidad de Buenos Aires en los años '30. Cuadernos de Filosofía, 40.

Vallina, C. (ed.) (2009). Crítica del testimonio. Ensayos sobre las relaciones entre memoria y relato. Rosario: Beatriz Viterbo.

Vedda, M. (comp.) (2008). Constelaciones dialécticas. Tentativas sobre Walter Benjamin. Bs. As.: Herramienta.

VVAA. (1993). Sobre Walter Benjamin. Vanguardias, historia, estética y literatura. Una visión latinoamericana. Buenos Aires: Alianza/Goethe-Institut.

Esta obra está licenciada com uma Licença Creative Commons Atribuição-NãoComercial-

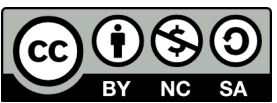

